\title{
Short-time kinetics and initial correlations in nonideal quantum systems
}

\author{
D.Kremp, D.Semkat, Th.Bornath \\ Universität Rostock, Institut für Physik, D-18051 Rostock, Germany \\ Received March 16, 2006, in final form May 12, 2006
}

\begin{abstract}
There are many reasons to consider quantum kinetic equations describing the evolution of a many-particle system on ultrashort time scales, e. g. correct energy conservation in nonideal plasmas, buildup of correlations (bound states), and kinetics of ultrafast processes in short-pulse laser plasmas.

We present a quantum kinetic theory including initial correlations and being valid on arbitrary time scales. Various examples of short-time relaxation processes, such as relaxation of the distribution function and the energy, are shown. Furthermore, gradient expansions of the general equations and the role of bound states are discussed.
\end{abstract}

Key words: kinetic theory, real-time Green's functions, short-time relaxation

PACS: 05.20.Dd, 52.25.Dg, 52.27.Gr

\section{Introduction}

Nonequilibrium properties of many-particle systems are successfully described by kinetic equations of the Boltzmann type. In the quantum case, the Boltzmann equation has the shape

$$
\left\{\frac{\partial}{\partial t}+\frac{\partial E_{a}}{\partial \mathbf{p}} \frac{\partial}{\partial \mathbf{R}}-\frac{\partial E_{a}}{\partial \mathbf{R}} \frac{\partial}{\partial \mathbf{p}}\right\} f_{a}(\mathbf{p R} t)=I_{a}(\mathbf{p R} T)
$$

where $f_{a}$ is the Wigner distribution. The most interesting term in this equation is the collision integral $I_{a}(\mathbf{p R} T)$ given here in binary collision approximation

$$
\begin{aligned}
I\left(\mathbf{p}_{1} \mathbf{R} T\right) & =\frac{2 \pi}{\hbar} \int \frac{\mathrm{d} \mathbf{p}_{2} \mathrm{~d} \overline{\mathbf{p}}_{1} \mathrm{~d} \overline{\mathbf{p}}_{2}}{(2 \pi \hbar)^{9}} \delta\left(E_{12}-\bar{E}_{12}\right)\left|\left\langle\mathbf{p}_{1} \mathbf{p}_{2}\left|T^{+}\left(E_{12}+\mathrm{i} \varepsilon\right)\right| \overline{\mathbf{p}}_{2} \overline{\mathbf{p}}_{1}\right\rangle\right|^{2} \\
& \times\left(\bar{f}_{1} \bar{f}_{2}\left(1 \pm f_{1}\right)\left(1 \pm f_{2}\right)-f_{1} f_{2}\left(1 \pm \bar{f}_{1}\right)\left(1 \pm \bar{f}_{2}\right)\right),
\end{aligned}
$$

and having the typical features of a Boltzmann-like collision integral: the kinetic energy conserving delta function, the transition probability in two-particle collisions given by the on-shell T-matrix and the usual combination of Wigner functions describing the occupation at the beginning and the end of the collision.

In spite of the fundamental character of Boltzmann-like kinetic equations, which describe the irreversible relaxation of an arbitrary initial Wigner distribution to the equilibrium distribution and, moreover, being the basic equations of transport theory, there exist many principle shortcomings. This was pointed out already by Prigogine [1] and Resibois [2], Kadanoff and Baym [3] and by Klimontovich [4]. The Boltzmann equation

1. cannot describe the short-time behavior $\left(t<t_{\text {corr }}\right)$ correctly,

2. conserves the kinetic energy $\langle T\rangle$ only instead of the total energy $\langle T\rangle+\langle V\rangle$ which is unphysical, especially for strongly correlated many-particle systems,

3. does not describe, because of the on-shell T-matrix, the influence of bound states on the kinetics,

4. gives as equilibrium solution the distribution for ideal particles. 
These shortcomings follow from a certain assumption necessary for the derivation of the Boltzmann equation from the basic equations of statistical mechanics. Let us consider this idea more in detail. The relaxation of a strongly correlated many-particle system from the initial state to the equilibrium state runs over several stages as shown in figure 1 . In the vicinity of the initial time, the evolution is strongly influenced by the initial condition, especially by the initial correlations.

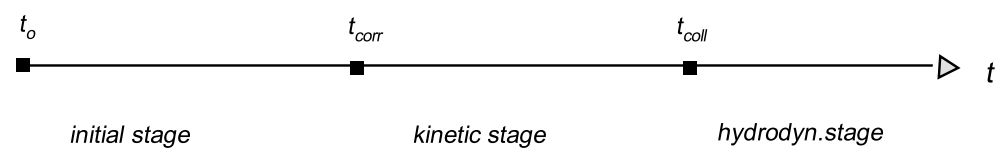

Figure 1. Stages of relaxation of a many-particle system.

We observe in this initial stage the buildup of correlations, and the kinetics is a non-Markovian one, i. e., there are memory effects. For times bigger than the correlation time ${ }^{1}$, the time evolution of the system is determined only by the one-particle density operator. All higher density operators are now functionals of the latter one. Initial correlation and memory effects are damped out, the kinetics is Markovian. Finally, after the collision time, we have local equilibrium and, therefore, a reduction of the phase space to the configuration space description.

Let us first consider a simple description of the relaxation process. We start from the Bogolyubov hierarchy for the reduced density operators in binary collision approximation. In this approximation there follows a closed system of equations

$$
\begin{aligned}
\mathrm{i} \hbar \frac{\mathrm{d} F_{1}}{\mathrm{~d} t}-\left[H_{1}^{H F}, F_{1}\right] & =n \operatorname{Tr}\left[V_{12}, g_{12}\right], \\
\mathrm{i} \hbar \frac{\mathrm{d} g_{12}}{\mathrm{~d} t}-\left[H_{12}^{H F}+V_{12}, g_{12}\right] & =\left[V_{12}, F_{1} F_{2}\right] .
\end{aligned}
$$

It is easy to find a formal solution of the second equation

$$
g_{12}(t)=U\left(t, t_{0}\right) g_{12}\left(t_{0}\right) U\left(t_{0}, t\right)+\frac{1}{\mathrm{i} \hbar} \int_{0}^{t-t_{o}} \mathrm{~d} \tau U(t, t-\tau)\left[V_{12}, F(t-\tau) F(t-\tau)\right] U(t-\tau, t) .
$$

Here, the first term describes the influence of the initial correlations on the time evolution of the system. The $U\left(t, t_{0}\right)$ are two-particle propagators. The second term describes the non-Markovian buildup of correlations, i. e., the value of the correlation operator at the time $t$ is determined by all previous times. Inserting now (5) into the first equation (3) leads to a closed non-Markovian equation for the one-particle density operator which describes the short-time behavior and the influence of initial correlations.

The Boltzmann equation follows from this equation under very restrictive assumptions:

i) We apply the Bogolyubov condition of weakening of initial correlations (no correct short-time behavior), i. e.

$$
\lim _{t_{0} \rightarrow-\infty} g_{12}\left(t_{0}\right)=0
$$

ii) We neglect the retardation (destruction of the correct energy conservation strongly influences the short-time behavior).

With condition (6), the initial stage is completely neglected. But there are many reasons to extend the description into the initial region. We here mention only, for example, correct energy conservation in nonideal plasmas, buildup of correlations, and kinetics of ultrafast processes, e. g. in short pulse laser plasmas.

\footnotetext{
${ }^{1}$ The correlation time can be defined as the duration of the interaction of two particles, i. e., the range of the interaction potential over the characteristic particle velocity (e. g. the thermal velocity). For plasmas, $t_{\text {corr }}$ is equal to the inverse plasma frequency.
} 
Of course the pure binary collision approximation is too simple, especially in describing the damping in the propagators $U$ and, therefore, the weakening of initial correlations and other many-particle effects like Pauli blocking, self-energy, screening of the Coulomb potential in plasmas, lowering of the ionization energy etc. A generalization of the equations $(3,4)$ is given in [5] and [6]. In this paper we shall consider these problems from a more general point of view of the powerful real-time Green's functions [3,7-10].

The original scheme of real-time Green's functions contains no contribution of initial correlations. In order to include them, several methods have been used, e. g. analytical continuation to real times which permits to include equilibrium initial correlations $[3,11,12]$, and perturbation theory with initial correlations $[13,14]$. A convincing solution has been presented by Danielewicz [8]. He developed a perturbation theory for a general initial state and derived generalized Kadanoff-Baym equations that take into account arbitrary initial correlations. A straightforward and very intuitive method which is not based on perturbation theory uses the equations of motion for the Green's functions [15-18]. It will be presented in the next sections.

Some effects of the initial stage can be taken into account already by expanding the nonMarkovian equation with respect to the time retardation and spatial nonlocality [1,3,19-21]. Therefore, we shall consider such an expansion in the last section of the paper in order to discuss the transition from the two-time Kadanoff-Baym equations (KBE) to the usual Markovian kinetic equation for the Wigner function.

\section{Martin-Schwinger hierarchy. Initial correlation}

The formalism of real-time Green's functions describes the many-particle system by a system of single-, two-, and more-particle Green's functions. We consider the definition of the single-particle Green's function as an average over the time-ordered product of field operators $\psi(1)$ and $\psi^{\dagger}\left(1^{\prime}\right)$,

$$
g\left(1,1^{\prime}\right)=\frac{\frac{1}{\mathrm{i} \hbar} \operatorname{Tr}\left\{\varrho \mathrm{T}_{\mathrm{C}}\left\{S\left(t_{0}, t_{0}\right) \psi(1) \psi^{\dagger}\left(1^{\prime}\right)\right\}\right\}}{\operatorname{Tr}\left\{\mathrm{T}_{\mathrm{C}} S\left(t_{0}, t_{0}\right)\right\}}
$$

with

$$
S\left(t_{0}, t_{0}\right)=\mathrm{T}_{\mathrm{C}} \exp \left\{-\mathrm{i} \int_{\mathrm{C}} \mathrm{d} 2 \mathrm{~d} \overline{2} U(2, \overline{2}) \psi^{\dagger}(2) \psi(\overline{2})\right\} .
$$

$\mathrm{T}_{\mathrm{C}}$ denotes the ordering along the Keldysh contour $[7,22]$. The field operators are given in the interaction picture with respect to a formal external potential $U\left(1,1^{\prime}\right)$. Their equation of motion is

$$
\left(\mathrm{i} \hbar \frac{\partial}{\partial t_{1}}+\frac{\hbar^{2} \nabla_{1}^{2}}{2 m}\right) \psi(1)= \pm\left.\int \mathrm{d} 2 V(1-2) \psi^{\dagger}(2) \psi(2) \psi(1)\right|_{t_{1}=t_{2}} .
$$

The density operator is in general a nonequilibrium one. In this case the adiabatic theorem is not valid and, therefore, we have a causal and anticausal time ordering. In order to handle this problem for nonequilibrium systems, the time ordering on the physical time axis has to be replaced by the ordering along the upper and lower branch of the Keldysh contour, see figure 2.

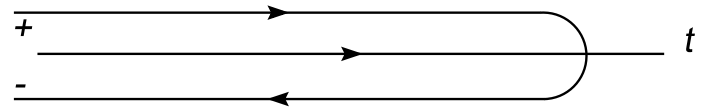

Figure 2. Keldysh time contour.

Now we introduce the following notation: times $t$ located on the upper $(+)$ branch are denoted by $t_{+}$, times located on the lower branch $(-)$by $t_{-}$. The expression $(7)$ is a very compact representation of the causal and anticausal single-particle Green's functions $g^{c(a)}\left(1,1^{\prime}\right)$ and the two correlation 
functions $g^{>(<)}\left(1,1^{\prime}\right)$. In dependence on the position of the two times $t$ and $t^{\prime}$ on the Keldysh contour, we get these functions by

$$
\begin{array}{ll}
g\left(1_{+}, 1_{+}^{\prime}\right)=g^{c}\left(1,1^{\prime}\right), & g\left(1_{-}, 1_{+}^{\prime}\right)=g^{>}\left(1,1^{\prime}\right), \\
g\left(1_{-}, 1_{-}^{\prime}\right)=g^{a}\left(1,1^{\prime}\right), & g\left(1_{+}, 1_{-}^{\prime}\right)=g^{<}\left(1,1^{\prime}\right) .
\end{array}
$$

Of special interest is the two-time correlation function $g^{<}\left(1,1^{\prime}\right)$. The time-diagonal part of this function is just the Wigner function.

In order to derive the equations of motion for the Green's functions (7) on the Keldysh contour, we have to introduce the equation of motion for the field operators (8) into the definition of the single-particle Green's function (7). Then follows immediately

$$
\int_{\mathcal{C}} \mathrm{d} \overline{1}\left\{g_{0}^{-1}(1, \overline{1})-U(1, \overline{1})\right\} g\left(\overline{1}, 1^{\prime} ; U\right)=\delta\left(1-1^{\prime}\right) \pm \mathrm{i} \hbar \int \mathrm{d} 2 V(1-2) g_{12}\left(12,1^{\prime} 2^{+} ; U\right)
$$

with

$$
g_{0}^{-1}\left(1,1^{\prime}\right)=\left(\mathrm{i} \hbar \frac{\partial}{\partial t_{1}}+\frac{\hbar^{2} \nabla_{1}^{2}}{2 m}\right) \delta\left(1-1^{\prime}\right)
$$

This equation is not closed. Due to the interaction between the particles, there is a coupling with the two-particle Green's function and so on. Equation (10) is therefore a member of an infinite chain of equations for many-particle Green's functions, well known as Martin-Schwinger hierarchy [23].

To find a closed equation for the one-particle Green's function, we proceed in well-known manner. First, we define the self-energy by

$$
\begin{aligned}
\int_{\mathcal{C}} \mathrm{d} \overline{1} \Sigma(1, \overline{1}) g\left(\overline{1}, 1^{\prime}\right) & = \pm \mathrm{i} \hbar \int \mathrm{d} 2 V(1-2) g_{12}\left(12,1^{\prime} 2^{+}\right) \\
& = \pm \mathrm{i} \hbar \int \mathrm{d} 2 V(1-2)\left\{ \pm \frac{\delta g\left(1,1^{\prime} ; U\right)}{\delta U\left(2^{+}, 2\right)}+g\left(1,1^{\prime}\right) g\left(2,2^{+}\right)\right\} .
\end{aligned}
$$

Here we took into account that $g_{12}$ can be derived from $g$ by means of functional derivation. Next, we introduce this definition into the first equation of the Martin-Schwinger hierarchy. Then follows immediately

$$
\left.\int_{\mathrm{C}} \mathrm{d} \overline{1}\left\{g_{0}^{-1}(1, \overline{1})-U(1, \overline{1})-\Sigma(1, \overline{1})\right)\right\} g\left(\overline{1}, 1^{\prime}\right)=\delta\left(1-1^{\prime}\right) .
$$

This equation is a Dyson equation for nonequilibrium systems which was derived for the first time by Kadanoff and Baym [3] and by Keldysh [7].

Of course, all problems are now transferred into the self-energy. Therefore, one has to consider the properties of this function and to find a possibility to determine appropriate approximations for $\Sigma$. Let us first consider the dependence of $\Sigma$ on the initial value of the two-particle Green's function. From (11) it is obvious that, in the limit $t \rightarrow t_{0}$, the self-energy has to fulfill the important relation

$$
\begin{aligned}
\lim _{t_{1}=t_{1}^{\prime}=t_{0}} \int_{\mathrm{C}} \mathrm{d} \overline{1} \Sigma(1, \overline{1}) g\left(\overline{1}, 1^{\prime}\right)= \pm \mathrm{i} \hbar \int \mathrm{d} \mathbf{r}_{2} V\left(\mathbf{r}_{1}-\mathbf{r}_{2}\right) g_{12}\left(\mathbf{r}_{1} \mathbf{r}_{2}, \mathbf{r}_{1}^{\prime} \mathbf{r}_{2} ; t_{0}\right) \\
\quad= \pm \mathrm{i} \hbar \int \mathrm{d} \mathbf{r}_{2} V\left(\mathbf{r}_{1}-\mathbf{r}_{2}\right)\left\{g\left(1,1^{\prime}\right) g\left(2,2^{+}\right)_{t_{0}} \pm g\left(1,2^{+}\right) g\left(2,1^{\prime}\right)_{t_{0}}+c\left(\mathbf{r}_{1} \mathbf{r}_{2}, \mathbf{r}_{1}^{\prime} \mathbf{r}_{2} ; t_{0}\right)\right\}
\end{aligned}
$$

which means that $\Sigma$ depends on an arbitrary initial value $g_{12}\left(\mathbf{r}_{1} \mathbf{r}_{2}, \mathbf{r}_{1}^{\prime} \mathbf{r}_{2} ; t_{0}\right)$ of the two-particle correlation function. Consequently, it is necessary to introduce initial conditions in order to determine the self-energy uniquely. However, for a regular integrand, the integral over the Keldysh contour vanishes in the limit $t, t^{\prime} \rightarrow t_{0}$. Therefore, equation (13) can be fulfilled only if the self-energy has a contribution proportional to a $\delta$-function with respect to the times. Such a term is the HartreeFock contribution $\Sigma^{\mathrm{HF}}$. But this term produces only the uncorrelated contribution to the initial 
binary density matrix. This means that the self-energy must contain a second part with a $\delta$-like singularity. The general structure of the self-energy is thus given by

$$
\begin{aligned}
\Sigma\left(1,1^{\prime}\right) & =\Sigma^{\mathrm{HF}}\left(1,1^{\prime}\right)+\Sigma^{c}\left(1,1^{\prime}\right)+\Sigma^{\mathrm{in}}\left(1,1^{\prime}\right), \\
\Sigma^{\mathrm{in}}\left(1,1^{\prime}\right) & =\Sigma^{\mathrm{in}}\left(1, \mathbf{r}_{1}^{\prime} t_{0}\right) \delta\left(t_{1}^{\prime}-t_{0}\right) .
\end{aligned}
$$

It has to be noted that in the adjoint Dyson equation there occurs the self-energy $\hat{\Sigma}$ having another initial contribution $\Sigma_{\text {in }}[17]$.

In order to discuss the influence of the initial correlations on the time evolution of the singleparticle Green's function, we insert (14) into equation (12) and fix the time arguments to opposite branches on the contour. Then we get the Kadanoff-Baym equations (KBE) for the correlation functions

$$
\begin{aligned}
& \left(\mathrm{i} \hbar \frac{\partial}{\partial t_{1}}+\frac{\hbar^{2} \nabla_{1}^{2}}{2 m}\right) g^{\gtrless}\left(1,1^{\prime}\right)-\int \mathrm{d} \overline{1} U(1, \overline{1}) g^{\gtrless}\left(\overline{1}, 1^{\prime}\right)=\int \mathrm{d} \bar{r}_{1} \Sigma^{\mathrm{HF}}(1, \overline{1}) g^{\gtrless}\left(\overline{1}, 1^{\prime}\right) \\
& \quad-\int_{t_{0}}^{t_{1}} \mathrm{~d} \overline{1}\left[\Sigma^{>}(1, \overline{1})-\Sigma^{<}(1, \overline{1})\right] g^{\gtrless}\left(\overline{1}, 1^{\prime}\right)+\int_{t_{0}}^{t_{1^{\prime}}} \mathrm{d} \overline{1}\left[\Sigma^{\gtrless}(1, \overline{1})+\Sigma^{\mathrm{in}}(1, \overline{1})\right]\left[g^{<}\left(\overline{1}, 1^{\prime}\right)-g^{>}\left(\overline{1}, 1^{\prime}\right)\right] .
\end{aligned}
$$

In contrast to the original KBE [3], there are two new properties: (i) the equations are valid for arbitrary initial times $t_{0}$, (ii) they contain an additional self-energy contribution $\Sigma^{\text {in }}$ which describes the influence of initial correlations. These equations are very general and lead, in the time-diagonal form and for special approximations for the self-energy, to all well-known standard kinetic equations for the Wigner distribution.

It is very useful to introduce retarded and advanced quantities by $F^{R / A}= \pm \Theta\left[ \pm\left(t-t^{\prime}\right)\right]$ $\left\{F^{>}\left(t, t^{\prime}\right)-F^{<}\left(t, t^{\prime}\right)\right\}$. Then the KBE take the convenient form

$$
\begin{gathered}
\left(\mathrm{i} \hbar \frac{\partial}{\partial t_{1}}+\frac{\hbar^{2} \nabla_{1}^{2}}{2 m}\right) g^{\gtrless}\left(1,1^{\prime}\right)-\int \mathrm{d} \overline{1} U(1, \overline{1}) g^{\gtrless}\left(\overline{1}, 1^{\prime}\right)-\int_{-\infty}^{\infty} \mathrm{d} \overline{1} \Sigma^{R}(1, \overline{1}) g^{\gtrless}\left(\overline{1}, 1^{\prime}\right) \\
=\int_{-\infty}^{\infty} \mathrm{d} \overline{1}\left[\Sigma^{\gtrless}(1, \overline{1})+\Sigma^{\mathrm{in}}(1, \overline{1})\right] g^{A}\left(\overline{1}, 1^{\prime}\right) \\
\left(\mathrm{i} \hbar \frac{\partial}{\partial t_{1}}+\frac{\hbar^{2} \nabla_{1}^{2}}{2 m}\right) g^{R / A}\left(1,1^{\prime}\right)-\int \mathrm{d} \overline{1} U(1, \overline{1}) g^{R / A}\left(\overline{1}, 1^{\prime}\right)-\int_{-\infty}^{\infty} \mathrm{d} \overline{1} \Sigma^{R / A}(1, \overline{1}) g^{R / A}\left(\overline{1}, 1^{\prime}\right)=\delta\left(1-1^{\prime}\right) .
\end{gathered}
$$

In order to determine the self-energy uniquely, boundary or initial conditions are necessary. Under the condition $t_{\text {corr }}<t_{\text {coll }}$ the Bogolyubov condition of weakening of initial correlations,

$$
\left.\lim _{t_{0} \rightarrow-\infty} g_{12}\left(12,1^{\prime} 2^{\prime}\right)\right|_{t_{0}}=\left[g\left(1,1^{\prime}\right) g\left(2,2^{\prime}\right) \pm g\left(1,2^{\prime}\right) g\left(2,1^{\prime}\right)\right]_{t_{0}},
$$

may be applied. With this condition follows $\Sigma^{\text {in }}=0$, and we get the original KBE. This was shown in $[15]$.

The most general and natural idea to fix the solution of the hierarchy for real times, however, is an initial condition

$$
\left.g_{12}\left(12,1^{\prime} 2^{\prime}\right)\right|_{t_{0}}=\left.g\left(1,1^{\prime}\right) g\left(2,2^{\prime}\right)\right|_{t_{0}} \pm\left. g\left(1,2^{\prime}\right) g\left(2,1^{\prime}\right)\right|_{t_{0}}+c\left(\mathbf{r}_{1} \mathbf{r}_{2}, \mathbf{r}_{1}^{\prime} \mathbf{r}_{2}^{\prime} ; t_{0}\right) .
$$

Here, $c\left(\mathbf{r}_{1} \mathbf{r}_{2}, \mathbf{r}_{1}^{\prime} \mathbf{r}_{2}^{\prime} ; t_{0}\right)$ is the initial correlation.

\section{Self-energy and initial correlations}

The central problem is to determine the self-energy including initial correlations. In order to find the self-energy function, we start with (11) and, therefore, need the functional derivative $\delta g / \delta U$. 
Fortunately, a simple procedure for the calculation of this quantity is available [3]. Equation (12) can be written for $t_{1}, t_{1}^{\prime}>t_{0}$ in the form

$$
\int_{\mathrm{C}} g^{-1}(1, \overline{1}) g\left(\overline{1}, 1^{\prime}\right) \mathrm{d} \overline{1}=\delta\left(1-1^{\prime}\right) .
$$

Here, the inverse Green's function $g^{-1}$ is given by

$$
g^{-1}(1, \overline{1})=g_{0}^{-1}(1, \overline{1})-U(1, \overline{1})-\Sigma(1, \overline{1}) .
$$

Functional differentiation of (19) with respect to the external potential yields

$$
\int_{\mathcal{C}} \mathrm{d} \overline{1} \frac{\delta g^{-1}(1, \overline{1})}{\delta U\left(2^{\prime}, 2\right)} g\left(\overline{1}, 1^{\prime}\right)=-\int_{\mathcal{C}} \mathrm{d} \overline{1} g^{-1}(1, \overline{1}) \frac{\delta g\left(\overline{1}, 1^{\prime}\right)}{\delta U\left(2^{\prime}, 2\right)} .
$$

Using (20), the general solution of this equation and its adjoint can be found:

$$
\begin{aligned}
\frac{\delta g\left(1,1^{\prime}\right)}{\delta U\left(2^{\prime}, 2\right)}= & \pm L\left(12,1^{\prime} 2^{\prime}\right)=g\left(1,2^{\prime}\right) g\left(2,1^{\prime}\right)+\int_{\mathcal{C}} \mathrm{d} \overline{1} \mathrm{~d} \overline{\overline{1}} g(1, \overline{1}) \frac{\delta\left[\Sigma(\overline{1}, \overline{\overline{1}})+\Sigma_{\text {in }}(\overline{1}, \overline{\overline{1}})\right]}{\delta U\left(2^{\prime}, 2\right)} g\left(\overline{\overline{1}}, 1^{\prime}\right) \\
& \pm C\left(12,1^{\prime} 2^{\prime}\right),
\end{aligned}
$$

where $C$ is an arbitrary function which obeys the homogeneous equation, i. e.

$$
\int_{\mathcal{C}} \mathrm{d} \overline{1} g^{-1}(1, \overline{1}) C\left(\overline{1} 2,1^{\prime} 2^{\prime}\right)=0
$$

and where $\Sigma_{\text {in }}$ is the initial correlation contribution to $\hat{\Sigma}$. There are three similar conditions to (23). One condition follows from the crossing symmetry $(1 \leftrightarrow 2)$ and two conditions follow from the adjoint equation of equation (12).

The function $C\left(12,1^{\prime} 2^{\prime}\right)$ makes it possible to take into account initial correlations. To show this we consider equation $(22)$ in the limit $t_{1}, t_{1}^{\prime} \rightarrow t_{0}$. Since the integral over the contour vanishes, it follows directly

$$
L\left(\mathbf{r}_{1}, \mathbf{r}_{2}, \mathbf{r}_{1}^{\prime}, \mathbf{r}_{2}^{\prime}, t_{0}\right)=c\left(\mathbf{r}_{1}, \mathbf{r}_{2}, \mathbf{r}_{1}^{\prime}, \mathbf{r}_{2}^{\prime}, t_{0}\right) \pm g\left(\mathbf{r}_{1}, \mathbf{r}_{2}^{\prime}, t_{0}\right) g\left(\mathbf{r}_{2}, \mathbf{r}_{1}^{\prime}, t_{0}\right) .
$$

Hence, the function $c\left(t_{0}\right)$ has to be identified with initial binary correlations.

In order to explore the temporal evolution of the function $C\left(12,1^{\prime} 2^{\prime}\right)$, we solve equation $(23)$ (and the three analogous relations) together with the initial condition

$$
\left.C\left(12,1^{\prime} 2^{\prime}\right)\right|_{t_{1}=t_{2}=t_{1}^{\prime}=t_{2}^{\prime}=t_{0}}=C\left(t_{0}\right) .
$$

The result is

$$
C\left(12,1^{\prime} 2^{\prime}\right)=\int_{\mathcal{C}} \mathrm{d} \overline{1} \mathrm{~d} \overline{2} \mathrm{~d} \overline{\overline{1}} \mathrm{~d} \overline{\overline{2}} g(1, \overline{1}) g(2, \overline{2}) c(\overline{1} \overline{2}, \overline{\overline{1}} \overline{\overline{2}}) g\left(\overline{\overline{2}}, 2^{\prime}\right) g\left(\overline{\overline{1}}, 1^{\prime}\right)
$$

with

$$
c(\overline{1} \overline{2}, \overline{\overline{1}} \overline{\overline{2}})=c\left(\overline{\mathbf{r}}_{1} t_{0}, \overline{\mathbf{r}}_{2} t_{0}, \overline{\mathbf{r}}_{1} t_{0}, \overline{\mathbf{r}}_{2} t_{0}\right) \delta\left(\bar{t}_{1}-t_{0}\right) \delta\left(\bar{t}_{2}-t_{0}\right) \delta\left(\overline{\bar{t}}_{1}-t_{0}\right) \delta\left(\overline{\bar{t}}_{2}-t_{0}\right) .
$$

For the self-energy $\Sigma$ we find, introducing equations (22) and (26) into (11), the following functional equation

$$
\begin{aligned}
\Sigma\left(1,1^{\prime}\right)= & \pm \mathrm{i} \hbar \int \mathrm{d} 2 V(1-2)\left\{ \pm \int_{\mathcal{C}} \mathrm{d} \overline{1} g(1, \overline{1}) \frac{\delta\left[\Sigma\left(\overline{1}, 1^{\prime}\right)+\Sigma_{\mathrm{in}}\left(\overline{1}, 1^{\prime}\right)\right]}{\delta U\left(2^{+}, 2\right)}+\delta\left(1-1^{\prime}\right) g\left(2,2^{+}\right)\right. \\
& \left. \pm \delta\left(2-1^{\prime}\right) g\left(1,2^{+}\right)+\int_{\mathcal{C}} \mathrm{d} \overline{1} \mathrm{~d} \overline{2} \mathrm{~d} \overline{\overline{2}} g(1, \overline{1}) g(2, \overline{2}) c\left(\overline{1} \overline{2}, 1^{\prime} \overline{\overline{2}}\right) g\left(\overline{\overline{2}}, 2^{+}\right)\right\} .
\end{aligned}
$$


For $\hat{\Sigma}$, a similar equation follows readily [17]. With equation (28), the self-energy is given as a functional of the interaction, the initial correlations, and the one-particle Green's function. From the definition of $c$, equation (27), it is obvious that the last contribution on the r.h.s. is local in time with a $\delta$-type singularity at $t=t^{\prime}$. Additional terms of this structure arise from the functional derivative. A further important property of the self-energy follows from comparing $\Sigma$, equation (28), with the corresponding expression for $\hat{\Sigma}$. One verifies that $\Sigma=\hat{\Sigma}$ for all times $t_{1}, t_{1}^{\prime}>t_{0}$, which means, in particular, that for these times, a well defined inverse Green's function does exist.

Equation (28) is well suited to derive approximations for the self-energy. By iteration, a perturbation series for $\Sigma$ in terms of $g, V$, and $c$ can be derived which begins with

$$
\begin{aligned}
\Sigma^{1}\left(1,1^{\prime}\right)= & \pm \mathrm{i} \hbar \delta\left(1-1^{\prime}\right) \int \mathrm{d} 2 V(1-2) g\left(2,2^{+}\right) \pm \mathrm{i} \hbar \int \mathrm{d} 2 V(1-2) \\
& \times \int_{\mathcal{C}} \mathrm{d} \overline{1} \mathrm{~d} \overline{2} \mathrm{~d} \overline{\overline{2}} g(1, \overline{1}) g(2, \overline{2}) c\left(\overline{1} \overline{2}, 1^{\prime} \overline{\overline{2}}\right) g\left(\overline{\overline{2}}, 2^{+}\right)+\text {exchange }
\end{aligned}
$$

or, in terms of Feynman diagrams,

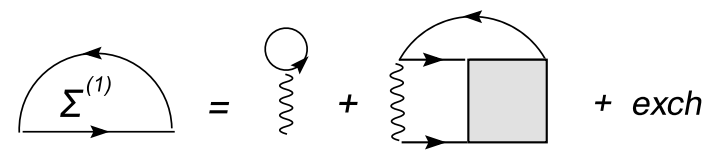

Second order contributions are evaluated straightforwardly, too, with the result

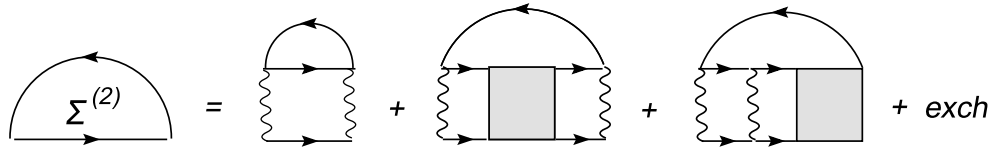

In contrast to the conventional diagram technique, the initial correlation appears now as a new basic element, drawn by a shaded rectangle. The analysis of the iteration scheme shows that all contributions to the self-energy (all diagrams) fall into two classes: (i) the terms $\Sigma^{\mathrm{HF}}$ und $\Sigma^{c}$ which begin and end with a potential, and (ii) $\Sigma^{\text {in }}$ beginning with a potential, but ending with an initial correlation. This is in accordance with the structure of the self-energy, equations (14), discussed in the previous section. The same result was obtained by Danielewicz based on his perturbation theory for general initial states [8].

If one considers the first two iterations for the self-energy more in detail, it becomes evident that in the initial correlation contribution - in front of the function $c$ - just the ladder terms appear which lead to the buildup of the two-particle Green's function. Obviously, the iteration "upgrades" the product of retarded one-particle propagators in the function $C$ to a full two-particle propagator, in the respective order, i. e., $\Sigma^{\text {in }}$ is of the form ${ }^{2}$

$$
\begin{aligned}
\Sigma^{\mathrm{in}}\left(1,1^{\prime}\right)= & \pm \mathrm{i} \hbar \int \mathrm{d} 2 V(1-2) \int \mathrm{d} \overline{\mathbf{r}}_{1} \mathrm{~d} \overline{\mathbf{r}}_{2} \mathrm{~d} \overline{\mathbf{r}}_{1} \mathrm{~d} \overline{\mathbf{r}}_{2} \\
& \times g_{12}^{R}\left(12, \overline{\mathbf{r}}_{1} t_{0}, \overline{\mathbf{r}}_{2} t_{0}\right) c\left(\overline{\mathbf{r}}_{1} t_{0}, \overline{\mathbf{r}}_{2} t_{0}, 1^{\prime}, \overline{\mathbf{r}}_{2} t_{0}\right) g^{A}\left(\overline{\overline{\mathbf{r}}}_{2} t_{0}, 2^{+}\right) \delta\left(t_{1}^{\prime}-t_{0}\right) .
\end{aligned}
$$

The analytical properties of $g_{12}^{R}\left(12, \overline{\mathbf{r}}_{1} t_{0}, \overline{\mathbf{r}}_{2} t_{0}\right)$ give rise to a damping $\gamma_{12}$ leading to a decay of the initial correlation for times $t>1 / \gamma_{12} \sim t_{\text {corr }}$. Thus, after the initial stage of relaxation, the Bogolyubov condition of weakening of initial correlations is reproduced automatically.

The method of including the initial binary correlations presented here is, undoubtedly, mathematically rigorous. The question arises which initial conditions are physically meaningful, i. e., which restrictions exist concerning the form of the function $c\left(t_{0}\right)$. The answer to that question has, in principle, to be given by the actual experimental situation. It can be shown, however, that a sufficient condition for the initial state is that it can be produced by a preceding evolution from another, in particular an uncorrelated, state, see [24]. The latter statement is again tangent to another question - why one does not include the description of such a preceding evolution instead

\footnotetext{
${ }^{2}$ Explicit expressions for $\Sigma$ and $\Sigma^{\text {in }}$ in T-matrix approximation have been given in [17].
} 
of constructing an initial correlation? The decision, of course, depends on the physical situation. However, there exist a variety of cases where the formation of the "initial" correlation is much more complicated than the result itself, consider, for example, the generation of a dense partially ionized plasma from a solid state target by an intense laser pulse. In such cases, the formalism developed above can be of high interest and usability.

\section{Numerical illustration and discussion}

Let us come back to the Kadanoff-Baym equations (15). Analytical solutions of these complex equations are available only for free particles. But also numerical solutions are possible up to now only for relatively simple approximations for the self-energy [25]. A short review of the numerical scheme for the solution of the Kadanoff-Baym equations and some results are given, e. g., in papers of Köhler et al. [26,27], and in the textbook [28].

Here we present the results for the numerical solution of the Kadanoff-Baym equations in second Born approximation for the self-energy. $V_{D}$ denotes the statically screened Coulomb potential. The initial correlation contribution is given by

$$
\begin{aligned}
\Sigma^{\mathrm{in}}\left(\mathbf{p}_{1} ; t_{1}, t_{1}^{\prime}\right)= & -\mathrm{i} \hbar^{5} \int \frac{\mathrm{d} \mathbf{p}_{2}}{(2 \pi \hbar)^{3}} \frac{\mathrm{d} \mathbf{q}}{(2 \pi \hbar)^{3}} V_{D}(\mathbf{q}) g^{R}\left(\mathbf{p}_{1}+\mathbf{q} ; t_{1}, t_{0}\right) g^{R}\left(\mathbf{p}_{2}-\mathbf{q} ; t_{1}, t_{0}\right) \\
& \times c\left(\mathbf{p}_{1}+\mathbf{q}, \mathbf{p}_{2}-\mathbf{q}, \mathbf{p}_{1}, \mathbf{p}_{2} ; t_{0}\right) g^{A}\left(\mathbf{p}_{2} ; t_{0}, t_{1}\right) \delta\left(t_{1}^{\prime}-t_{0}\right) .
\end{aligned}
$$

The initial correlations were chosen in the form of the Debye pair correlation:

$$
c\left(\mathbf{p}_{1}, \mathbf{p}_{2} ; \mathbf{p}_{1}+\mathbf{q}, \mathbf{p}_{2}-\mathbf{q} ; t_{0}\right)=-\left.\frac{1}{(\mathrm{i} \hbar)^{2}} \frac{V_{D}(\mathbf{q})}{k_{\mathrm{B}} T} f\left(\mathbf{p}_{1}\right) f\left(\mathbf{p}_{2}\right)\left[1-f\left(\mathbf{p}_{1}+\mathbf{q}\right)\right]\left[1-f\left(\mathbf{p}_{2}-\mathbf{q}\right)\right]\right|_{t_{0}} .
$$

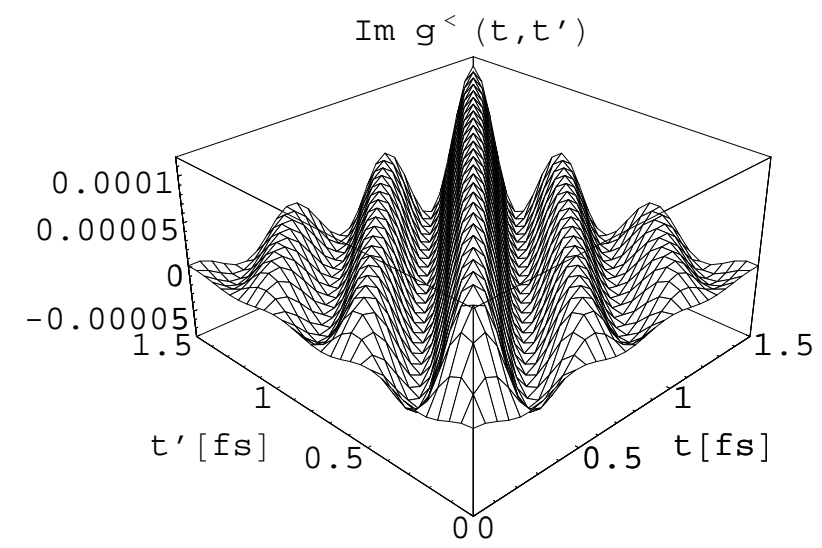

Figure 3. Temporal evolution of the imaginary part of $g^{<}\left(t, t^{\prime}\right)$, momentum $k=0.8 / a_{B}$, for electrons in hydrogen. The initial distribution is a Fermi distribution with $T=10000 \mathrm{~K}$ and $n=10^{21} \mathrm{~cm}^{-3}$.

As a model system, electrons in a hydrogen plasma are considered with the density $n=10^{21}$ $\mathrm{cm}^{-3}$ and the initial temperature $T_{0}=10000 \mathrm{~K}$. Figure 3 shows the result of the numerical computation for the full time dependence of $\operatorname{Im} g^{<}\left(\mathbf{p}, t, t^{\prime}\right)$ for a fixed momentum. The time evolution of the Wigner function for this fixed momentum is given on the time diagonal $t=t^{\prime}$. Perpendicular to the diagonal, the spectral properties become apparent. There are oscillations which are connected with the single-particle energy and a damping of the amplitude which characterizes the damping of the single-particle states.

Macrophysical quantities are directly computed from the correlation function. For example, the kinetic energy is given by

$$
\langle T\rangle(t)=\mathcal{V} \int \frac{\mathrm{d} \mathbf{p}}{(2 \pi \hbar)^{3}} \frac{p^{2}}{2 m}(-\mathrm{i} \hbar) g^{<}(\mathbf{p}, t, t)
$$


and the potential energy follows from

$$
\langle V\rangle(t)=\frac{1}{4} \mathcal{V} \int \frac{\mathrm{d} \mathbf{p}}{(2 \pi \hbar)^{3}}\left\{\left(\mathrm{i} \hbar \frac{\partial}{\partial t}-\mathrm{i} \hbar \frac{\partial}{\partial t^{\prime}}\right)-\frac{p^{2}}{m}\right\}(-\mathrm{i} \hbar) g^{<}\left(\mathbf{p}, t, t^{\prime}\right)_{t=t^{\prime}}
$$

Here, $\mathcal{V}$ is the system volume.

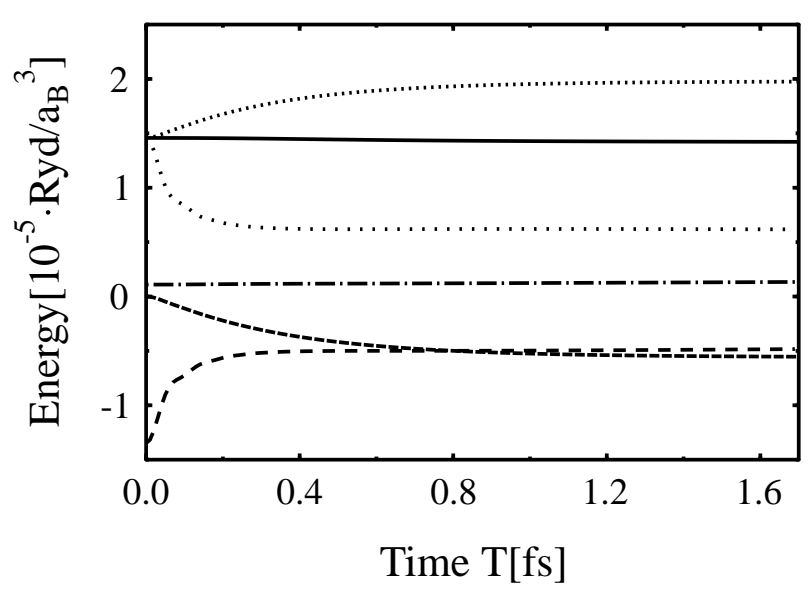

Figure 4. Temporal evolution of the kinetic energy without (narrow-dotted line) and with initial correlations (wide-dotted line), potential energy w/o (narrow-dashed line) and with initial correlations (wide-dashed line) and total energy without (solid line) and with initial correlations (dash-dotted line) for electrons in a hydrogen plasma. Parameters see in figure 3.

Figure 4 shows the time evolution of kinetic, potential, and total energies for electrons in a hydrogen plasma for two different initial states: an initially correlated and an uncorrelated case [18]. The following analogies and differences can be observed:

- In spite of the same initial density and temperature, the initial states are principally different. In the initially uncorrelated case, the correlation energy is zero at $t_{0}=0$. If there exist initial correlations, it has already a finite value, leading, e. g., to a different total energy.

- The behavior for short times $\left(t<t_{\text {corr }}\right)$ is completely different, too. In both cases, this stage is determined by the correlation buildup. In the case of nonzero initial correlations, however, this buildup is superposed by their decay. Therefore, the value of the correlation energy is decreased.

- The evolution in the kinetic stage, i. e. after the correlation time $t_{\text {corr }}$, is independent of the initial correlation. Thus, Bogolyubov's weakening condition [cf. equation (6)] comes out automatically in that stage without postulating it, i. e., the system has "forgotten" about the initial state.

- Due to total energy conservation, different final kinetic energies (and therefore, final temperatures) are reached. Thus, the equilibrium state is effected by the initial correlation due to its contribution to the total energy.

- The equilibrium correlations agree approximately. They are nearly independent of the initial state.

We can conclude that the energy evolution on short time scales strongly depends on the initial state of the system, especially on the initial binary correlations. In the example shown above, the initial state is overcorrelated and, thus, causes a reduction of the kinetic energy. It can be easily verified by a simple energy balance that an overcorrelated initial state will always cause a decrease of kinetic energy while an undercorrelated initial state leads to its increase. Apart from contributions due to correlations built up in the system, the kinetic energy is closely connected 
with the temperature. Thus, we can conclude that, by preparing an overcorrelated initial state, it should be possible to cool down a system [29-31]. This cooling effect can be of experimental importance e. g. for ultracold ions in traps in order to reach still lower temperatures.

\section{Kinetic equation in first order gradient expansion. Bound states}

Let us now discuss the transition from the Kadanoff-Baym equations (KBE) to the usual kinetic equations for the Wigner function,

$$
f(\mathbf{p}, \mathbf{R}, t)=\int \frac{\mathrm{d} \omega}{2 \pi}( \pm \mathrm{i}) g^{<}(\mathbf{p}, \omega, \mathbf{R}, t),
$$

with $g^{<}(\mathbf{p}, \omega, \mathbf{R}, T)$ being the Fourier transform of $g^{<}\left(1,1^{\prime}\right)=g^{<}(\mathbf{r}, t, \mathbf{R}, T)$ with respect to the variables $\mathbf{r}=\mathbf{r}_{1}-\mathbf{r}_{1}^{\prime}$ and $t=t_{1}-t_{1}^{\prime}$, respectively. $\mathbf{R}$ and $\mathrm{T}$ are determined by $\mathbf{R}=\left(\mathbf{r}_{1}+\mathbf{r}_{1}^{\prime}\right) / 2$ and $T=\left(t_{1}+t_{1}^{\prime}\right) / 2$.

An equation for the Wigner function follows from (15) and its adjoint equation in the time diagonal case $t_{1}=t_{1}^{\prime}=T$,

$$
\begin{aligned}
\left(\frac{\partial}{\partial T}\right. & \left.+\frac{\mathbf{p}}{m_{a}} \nabla_{\mathbf{R}}-\nabla_{\mathbf{R}} U_{a}^{\mathrm{eff}}(\mathbf{R}, t) \nabla_{\mathbf{p}}\right) f_{a}(\mathbf{p}, \mathbf{R}, T)=\int_{t_{0}}^{T} \mathrm{~d} \bar{t}\left\{\left[\Sigma^{<}(T, \bar{t})+\Sigma^{\mathrm{in}}(T, \bar{t})\right]\right. \\
& \left.\times g^{A}(\bar{t}, T)+\Sigma^{R}(T, \bar{t}) g^{<}(\bar{t}, T)-g^{<}(T, \bar{t}) \Sigma^{A}(\bar{t}, T)-g^{R}(T, \bar{t})\left[\Sigma^{<}(\bar{t}, T)+\Sigma_{\mathrm{in}}(\bar{t}, T)\right]\right\} .
\end{aligned}
$$

The time-diagonal KBE is the most general quantum kinetic equation. Like in the case of the two-time KBE, we retained still (i) the effect of initial correlations, (ii) the effect of retardation, and (iii) the validity of conservation laws. The r.h.s. of equation (36) represents a far-reaching generalization of usual collision integrals. However, the kinetic equation for the Wigner function is not closed because the single-particle Green's function arises on the r.h.s. In order to get a closed kinetic equation in explicit form, one still has to solve two problems:

(i) The self energy $\Sigma$ has to be determined as a functional of $g \gtrless$ by appropriate approximations. Standard approximations are, e. g., the T-matrix approximation and the RPA.

(ii) The two-time correlation functions have to be reconstructed from the Wigner distribution. This so-called reconstruction problem is often solved using the Kadanoff-Baym ansatz (KBA). A more general solution was proposed by Lipavský, Špička, and Velický [32] who derived an integral equation for the reconstruction. Usually only the first iteration of this equation, the generalized Kadanoff-Baym ansatz (GKBA)

$$
\pm g^{<}\left(t_{1}, t_{1}^{\prime}\right)=g^{R}\left(t_{1}, t_{1}^{\prime}\right) f\left(t_{1}^{\prime}\right)-f\left(t_{1}\right) g^{A}\left(t_{1}, t_{1}^{\prime}\right)
$$

is used. With the GKBA one gets from (36) a closed non-Markovian kinetic equation for the Wigner distribution $[5,6,10,17]$. We shall not follow this line here.

Some effects of the initial stage can be taken into account already by expansions with respect to the retardation. The KBE (16) are nonlocal equations in space and time. Applying the gradient expansion $[3,9,10]$ to $(16)$ and the adjoint equation, one obtains for the correlation functions $g \gtrless$ the following equations

$$
\left\{\operatorname{Re} g^{R^{-1}}, \mathrm{i} g^{\gtrless}\right\}-\left\{\mathrm{i} \Sigma^{\gtrless}, \operatorname{Re} g^{R}\right\}=g^{<\Sigma^{>}}-g^{>} \Sigma^{<} .
$$

For the corresponding spectral function $a=\mathrm{i}\left(g^{>}-g^{<}\right)=\mathrm{i}\left(g^{R}-g^{A}\right)$ the difference of the two above equations gives

$$
\left\{\operatorname{Re} g^{R^{-1}}, a\right\}-\left\{\Gamma, \operatorname{Re} g^{R}\right\}=0 .
$$


All quantities in (38) and (39) are functions of $\mathbf{p}, \omega, \mathbf{R}, T$. The damping $\Gamma=\mathrm{i}\left(\Sigma^{>}-\Sigma^{<}\right)=-2 \operatorname{Im} \Sigma^{\mathrm{R}}$ was introduced, and Poisson brackets were defined by

$$
\{A, B\}=\frac{\partial A}{\partial \omega} \frac{\partial B}{\partial T}-\frac{\partial A}{\partial T} \frac{\partial B}{\partial \omega}-\frac{\partial A}{\partial \mathbf{p}} \frac{\partial B}{\partial \mathbf{R}}+\frac{\partial A}{\partial \mathbf{R}} \frac{\partial B}{\partial \mathbf{p}} .
$$

The equations for the retarded and advanced Green's functions on the same level of approximation read

$$
\left(\omega \pm \mathrm{i} \varepsilon-\frac{p^{2}}{2 m}-\Sigma^{R / A}(\mathbf{p}, \omega, \mathbf{R}, T)\right) g^{R / A}(\mathbf{p}, \omega, \mathbf{R}, T)=1 .
$$

The KBE (38) in gradient approximation are the basic equations for our further consideration. We shall follow the line of the papers $[21,33,34]$. The first bracket on the l.h.s. of (38) is a general drift term, and the r.h.s. describes collisions. The second contribution on the l.h.s. of equation (38) is not so easy to interprete. If damping is small, it may be neglected, cf. (39). In general, this term is off-shell with respect to quasiparticle energies.

If this term is neglected - no damping - one gets with $\operatorname{Re} g^{R^{-1}}=g_{0}^{-1}-\Sigma^{H F}-\operatorname{Re} \Sigma_{\text {corr }}^{R}$ for the spectral function the following expression

$$
a(\omega, p)=2 \pi \delta\left(\omega-p^{2} / 2 m-\operatorname{Re} \Sigma^{R}\right)=Z 2 \pi \delta(\omega-E(p))
$$

with the quasiparticle energy $E(p)=p^{2} / 2 m+\operatorname{Re} \Sigma^{R}(\omega=E)$ and the renormalization factor

$$
Z^{-1}=1-\left.\frac{\partial \operatorname{Re} \Sigma^{R}}{\partial \omega}\right|_{\omega=E} .
$$

The drift term in the corresponding kinetic equation is called, therefore, on-shell drift. The different approximations for the on-shell drift are connected with the assumption of the smallness of the gradient contributions. In dependence on the approximation of $\operatorname{Re} g^{R^{-1}}$, the free particle, the mean field and the quasiparticle drifts may be obtained as it was carried out in detail in [35]. The resulting kinetic equation describes ideal (quasi)particles.

Retaining the off-shell contribution, however, enables us to overcome the shortcomings of that equation, cf. the discussion of Boltzmann-like equations (1) in the introduction. One is able to find nonideality contributions and to avoid inconsistencies like violation of sum rules by (42) etc. The derivatives on the l.h.s. can be rearranged to give a more useful form

$$
\frac{\partial}{\partial T}\left[\mathrm{i} g^{\gtrless}-\frac{\partial \operatorname{Re} \Sigma^{R}}{\partial \omega} \mathrm{i} g^{\gtrless}+\mathrm{i} \Sigma \gtrless \frac{\partial \operatorname{Re} g^{R}}{\partial \omega}\right]+\frac{\partial}{\partial \omega}\left[\frac{\partial \operatorname{Re} \Sigma^{R}}{\partial T} \mathrm{i} g^{\gtrless}-\mathrm{i} \Sigma \gtrless \frac{\partial \operatorname{Re} g^{R}}{\partial T}\right]=g^{<} \Sigma^{>}-g^{>} \Sigma^{<} .
$$

For the sake of clarity, we dropped the derivatives with respect to $\mathbf{p}$ and $\mathbf{R}$.

A kinetic equation for the Wigner function (35) follows immediately by an integration of (44) with respect to $\omega$

$$
\frac{\partial f}{\partial T}-\frac{\partial}{\partial T} \int \frac{\mathrm{d} \omega}{2 \pi}\left[( \pm \mathrm{i}) g^{<} \frac{\partial \operatorname{Re} \Sigma^{R}}{\partial \omega}-( \pm \mathrm{i}) \Sigma^{<} \frac{\partial \operatorname{Re} g^{R}}{\partial \omega}\right]= \pm \int \frac{\mathrm{d} \omega}{2 \pi}\left(g^{<} \Sigma^{>}-g^{>} \Sigma^{<}\right) .
$$

Using the dispersion relations for $\operatorname{Re} g$ and $\operatorname{Re} \Sigma$, this equation can be transformed to

$$
\frac{\partial f}{\partial T}=I^{0}(p, T)+I^{1}(p, T)
$$

with

$$
\begin{aligned}
I^{0}\left(p_{1} T\right) & =\int \frac{\mathrm{d} \omega}{2 \pi}\left[( \pm \mathrm{i}) \Sigma^{<}(\omega T) \mathrm{i} g^{>}(\omega T)-\mathrm{i} \Sigma^{>}(\omega T)( \pm \mathrm{i}) g^{<}(\omega T)\right] \\
I^{1}\left(p_{1} T\right) & =\frac{\partial}{\partial T} \int \frac{\mathrm{d} \omega_{1} \mathrm{~d} \omega_{2}}{(2 \pi)^{2}} \frac{P^{\prime}}{\omega_{1}-\omega_{2}}\left[\mathrm{i} \Sigma^{>}\left(\omega_{1} T\right)( \pm \mathrm{i}) g^{<}\left(\omega_{2} T\right)-( \pm \mathrm{i}) \Sigma^{<}\left(\omega_{1} T\right) \mathrm{i} g^{>}\left(\omega_{2} T\right)\right]
\end{aligned}
$$


Equation (46) represents a kinetic equation for the full Wigner distribution function. On the r.h.s., the collision integrals are given in terms of the correlation functions $g \gtrless$ and the self-energy functions $\Sigma \gtrless[g \gtrless]$. The latter are, again, functionals of the $g \gtrless$. Therefore, a reconstruction formula is needed in $I^{0}$ and $I^{1}$ in order to get a closed kinetic equation for the Wigner distribution function $f$. The additional collision integral $I^{1}$ in (46) is clearly a new element and reflects nonideality corrections. However, also the term $I^{0}$ contains in the self-energy functions further gradient (nonideality) corrections besides the usual Boltzmann-like terms.

For further progress in deriving a kinetic equation, the connection between the correlation functions $g \gtrless$ and the Wigner distribution will be investigated. The starting point is the equation (44). The real part of the retarded Green's function can be determined using the dispersion relation. In the limit $\Gamma \rightarrow 0$ it follows that

$$
\operatorname{Re} g^{R}(\mathbf{p}, \omega, \mathbf{R}, T)=\frac{P}{\omega-\frac{p^{2}}{2 m}-\operatorname{Re} \Sigma^{R}(\mathbf{p}, \omega, \mathbf{R}, T)} .
$$

Taking this approximation for $\operatorname{Re} g^{R}$ in equation (44), one gets

$$
\begin{aligned}
\frac{\partial}{\partial T}[ & \left.\left(1-\frac{\partial \operatorname{Re} \Sigma^{R}}{\partial \omega}\right)\left(\mathrm{i} g^{\gtrless}+\mathrm{i} \Sigma^{\gtrless} \frac{P^{\prime}}{\omega-\frac{p^{2}}{2 m}-\operatorname{Re} \Sigma^{R}}\right)\right] \\
& +\frac{\partial}{\partial \omega}\left[\frac{\partial \operatorname{Re} \Sigma^{R}}{\partial T}\left(\mathrm{i} g^{\gtrless}+\mathrm{i} \Sigma^{\gtrless} \frac{P^{\prime}}{\omega-\frac{p^{2}}{2 m}-\operatorname{Re} \Sigma^{R}}\right)\right]=g^{<} \Sigma^{>}-g^{>} \Sigma^{<} .
\end{aligned}
$$

The structure of this equation now suggests to define a new quantity $Q^{\gtrless}$ by

$$
\mathrm{i} Q^{\gtrless}=\mathrm{i} g^{\gtrless}+\mathrm{i} \Sigma \gtrless \frac{P^{\prime}}{\omega-\frac{p^{2}}{2 m}-\operatorname{Re} \Sigma^{R}},
$$

which leads to a kinetic equation for the $Q^{\gtrless}$

$$
\frac{\partial}{\partial T}\left[\left(1-\frac{\partial \operatorname{Re} \Sigma}{\partial \omega}\right) \mathrm{i} Q^{\gtrless}\right]+\frac{\partial}{\partial \omega}\left[\frac{\partial \operatorname{Re} \Sigma}{\partial T} \mathrm{i} Q^{\gtrless}\right]=Q^{<\Sigma^{>}}-Q^{>} \Sigma^{<} .
$$

The physical meaning of the special correlation functions $Q^{\gtrless}$ becomes obvious if we introduce a spectral function according to the usual definition by $A_{Q}=\mathrm{i}\left(Q^{>}-Q^{<}\right)$. The equation for $A_{Q}$ following from (52)

$$
\frac{\partial}{\partial T}\left[\left(1-\frac{\partial \operatorname{Re} \Sigma}{\partial \omega}\right) \mathrm{i}\left(Q^{>}-Q^{<}\right)\right]+\frac{\partial}{\partial \omega}\left[\frac{\partial \operatorname{Re} \Sigma}{\partial T} \mathrm{i}\left(Q^{>}-Q^{<}\right)\right]=0
$$

has the solution

$$
A_{Q}=i\left(Q^{>}-Q^{<}\right)=2 \pi \delta\left(\omega-\frac{p^{2}}{2 m}-\operatorname{Re} \Sigma^{R}\right)
$$

From the representation (53), it turns out that the quantity $Q$ describes quasiparticles $[21,33,34]$.

It is useful to introduce a quasiparticle distribution function by

$$
\begin{aligned}
\pm \mathrm{i} Q^{<} & =2 \pi \delta\left(\omega-\frac{p^{2}}{2 m}-\operatorname{Re} \Sigma^{R}\right) f^{Q}(\mathbf{p}, \mathbf{R}, T) \pm X(\mathbf{p}, \omega, \mathbf{R}, T) \\
\mathrm{i} Q^{>} & =2 \pi \delta\left(\omega-\frac{p^{2}}{2 m}-\operatorname{Re} \Sigma^{R}\right)\left(1 \pm f^{Q}(\mathbf{p}, \mathbf{R}, T)\right)+X(\mathbf{p}, \omega, \mathbf{R}, T)
\end{aligned}
$$

with a function $X(\mathbf{p}, \omega, \mathbf{R}, T)$ arbitrary so far.

The determination of the quantity $X$ is the point where the problem of an ansatz arises. As stated above, the correlation functions $Q^{\gtrless}$ describe undamped quasiparticles. If these functions 
are considered in the time domain, an ansatz similar to the generalized Kadanoff-Baym ansatz can be used:

$$
\pm Q^{<}\left(t, t^{\prime}\right)=G_{Q}^{R}\left(t, t^{\prime}\right) f^{Q}\left(t^{\prime}\right)-f^{Q}(t) G_{Q}^{A}\left(t, t^{\prime}\right)
$$

with $f^{Q}$ and $G_{Q}^{R / A}$ being the quasiparticle distribution function and the quasiparticle popagators, respectively. Introducing the difference time $\tau$ and the centered time $T$ in the usual way and performing an expansion with respect to the retardation in the quasiparticle distribution, we get after Fourier transformation

$$
\pm \mathrm{i} Q^{<}(\omega, T)=A_{Q}(\omega, T) f^{Q}(T)-\frac{\partial}{\partial \omega} \operatorname{Re} G_{Q}^{R}(\omega, T) \frac{\partial}{\partial T} f^{Q}(T) .
$$

The correlation function $Q^{>}$is given in a similar way by

$$
\mathrm{i} Q^{>}(\omega, T)=A_{Q}(\omega, T)\left(1 \pm f^{Q}(T)\right)-\frac{\partial}{\partial \omega} \operatorname{Re} G_{Q}^{R}(\omega, T) \frac{\partial}{\partial T}\left(1 \pm f^{Q}(T)\right)
$$

Comparison with $(54,55)$ gives

$$
X(\omega, T)=-\frac{\partial}{\partial \omega} \operatorname{Re} G_{Q}^{R}(\omega, T) \frac{\partial}{\partial T}\left( \pm f^{Q}(T)\right) .
$$

If we insert (57) in (51), the following expression in terms of the quasiparticle distribution function can be derived for $g^{<}[21]$

$$
\begin{aligned}
\pm \mathrm{i} g^{<}(\omega, T)= & 2 \pi \delta\left(\omega-\frac{p^{2}}{2 m}-\operatorname{Re} \Sigma^{R}\right) f^{Q}(T)-\frac{P^{\prime}}{\left(\omega-\frac{p^{2}}{2 m}-\operatorname{Re} \Sigma\right)}( \pm \mathrm{i}) \Sigma^{<}(\omega, T) \\
& -\frac{P^{\prime}}{\omega-\frac{p^{2}}{2 m}-\operatorname{Re} \Sigma(\omega)} \frac{\partial}{\partial T}\left( \pm f^{Q}(T)\right) .
\end{aligned}
$$

A similar expression, but without the last term on the r.h.s was found in [33,34], see also [35]. As it can be seen from this expression, the correlation functions consist of a pole contribution and an off-pole part.

In order to get an equation for $f^{Q}$, one can use equation (52) together with (60). As the quantity $X$ (59) is of first order in the derivatives (it consists of a collision term), it can be neglected on the l.h.s. of (52). The $\delta$-function in (60) leads to the quasiparticle energies. Then $\operatorname{Re} \Sigma$ does not only depend on the variables $\mathbf{R}$ or $\mathbf{p}$ explicitly but also implicitly via $\omega=E(\mathbf{p}, \mathbf{R}, T)[3]$. With an integration with respect to $\omega$ and the reconstruction formulae (57) and (58) there follows a kinetic equation for determining the quasiparticle's distribution function

$$
\begin{aligned}
{\left[\frac{\partial}{\partial T}+\frac{\partial E}{\partial \mathbf{p}} \frac{\partial}{\partial \mathbf{R}}-\frac{\partial E}{\partial \mathbf{R}} \frac{\partial}{\partial \mathbf{p}}\right] f^{Q}(\mathbf{p}, \mathbf{R}, T)=} & Z\left[( \pm \mathrm{i}) \Sigma^{<}(\mathbf{p}, E, \mathbf{R}, T)\left(1 \pm f^{Q}(\mathbf{p}, \mathbf{R}, T)\right)\right. \\
& \left.-\mathrm{i} \Sigma^{>}(\mathbf{p}, E, \mathbf{R}, T) f^{Q}(\mathbf{p}, \mathbf{R}, T)\right] .
\end{aligned}
$$

This equation is well known as Landau-Silin equation in the theory of Fermi liquids.

Now it is easy to obtain the full Wigner function. Integration of (60) with respect to $\omega$ and expansion of the renormalization factor $Z$ gives $[21,35]$

$$
\begin{aligned}
f(\mathbf{p}, \mathbf{R}, T)= & f^{Q}(\mathbf{p}, \mathbf{R}, T)\left(1+\frac{\partial}{\partial \omega} \operatorname{Re} \Sigma(\mathbf{p}, \omega, \mathbf{R}, T)_{\mid \omega=E}\right) \\
& -\int \frac{\mathrm{d} \omega}{2 \pi} \frac{P^{\prime}}{\left(\omega-\frac{p^{2}}{2 m}-\operatorname{Re} \Sigma\right)}( \pm \mathrm{i}) \Sigma^{<}(\omega, T) .
\end{aligned}
$$

With equation (62), an interesting relation was obtained which makes it possible to determine the Wigner function $f$ from the quasiparticle distribution function $f^{Q}$ which may be obtained from the 
Landau-Silin equation. The first term on the r.h.s. describes ideal quasiparticles and the second one stands for the scattering contribution of quasiparticles.

Finally we have to express the correlation function $g \gtrless$ in terms of the Wigner function $f$ instead of the quasiparticle distribution $f^{Q}$. This can be done in the way that the quasiparticle distribution function in (60) is substituted by the full Wigner function using equation (62) [21]

$$
\begin{aligned}
\pm \mathrm{i} g^{<}(\omega, T)= & 2 \pi \delta(\omega-E) f(T)-\frac{P^{\prime}}{\omega-E} \frac{\partial}{\partial T} f(T) \\
& -\frac{P^{\prime}}{(\omega-E)}( \pm \mathrm{i}) \Sigma^{<}(\omega, T)+2 \pi \delta(\omega-E) \int \frac{\mathrm{d} \bar{\omega}}{2 \pi} \frac{P^{\prime}}{(\bar{\omega}-E)}( \pm \mathrm{i}) \Sigma^{<}(\bar{\omega}, T) .
\end{aligned}
$$

In the case of the thermodynamic equilibrium, $\Sigma^{<}=\Gamma f(\omega)$ with $f(\omega)$ being Bose- or Fermi-like functions. Then from (63) the extended quasiparticle approximation follows [10,36].

Now we are able to find a closed kinetic equation for the Wigner function in first order gradient approximation. We start with equation (48). On the r.h.s., the collision integrals are given in terms of the one particle correlation functions $g \gtrless$ and the self-energy functions $\Sigma \gtrless$. The latter are, again, functionals of the $g \gtrless$. As an example we consider the self-energy in a ladder approximation, i. e., it is given by the T-matrix $T^{\gtrless}$

$$
\Sigma \gtrless\left(\mathbf{p}_{1} \omega, T\right)=\int \frac{\mathrm{d} \mathbf{p}_{2}}{(2 \pi)^{3}} \frac{\mathrm{d} \bar{\omega}}{2 \pi}\left\langle\mathbf{p}_{1} \mathbf{p}_{2}\left|T^{\gtrless}(\omega+\bar{\omega}, T)\right| \mathbf{p}_{2} \mathbf{p}_{1}\right\rangle( \pm \mathrm{i}) g^{\lessgtr}\left(\mathbf{p}_{2} \bar{\omega}, T\right) .
$$

Expressing the correlation functions of the T-matrix via the so-called optical theorem, however, leads to further gradient terms. Using further the reconstruction formula (63) in $I^{0}$ and $I^{1}$ to the appropriate order, one gets a kinetic equation for the Wigner distribution function $f$. We obtain the Boltzmann equation in first order retardation $[5,21,37]$

$$
\begin{aligned}
\left(\frac{\partial}{\partial T}+\frac{\mathbf{p}}{m_{a}} \nabla_{\mathbf{R}}-\nabla_{\mathbf{R}} U_{a}^{\mathrm{eff}}(\mathbf{R}, T) \nabla_{\mathbf{p}}\right) f_{a}(\mathbf{p}, \mathbf{R}, T) & =\lim _{\varepsilon \rightarrow 0}\left(1+\frac{1}{2} \frac{\mathrm{d}}{\mathrm{d} T} \frac{\mathrm{d}}{\mathrm{d} \varepsilon}\right) I^{B}(\mathbf{p}, \varepsilon) \\
& =I^{B}(\mathbf{p})+I^{R}(\mathbf{p}) .
\end{aligned}
$$

Here, $\left.I^{B}(\mathbf{p}, \varepsilon)\right|_{\varepsilon \rightarrow 0}=I^{B}(\mathbf{p})$ is the Boltzmann collision integral with

$$
\begin{aligned}
I^{B}(\varepsilon)= & -\int \mathrm{d} \mathbf{p}_{2} \mathrm{~d} \overline{\mathbf{p}}_{1} \mathrm{~d} \overline{\mathbf{p}}_{2} \int \mathrm{d} \omega \frac{1}{\pi} \frac{\varepsilon}{\left(\omega-E_{12}\right)^{2}+\varepsilon^{2}} \frac{1}{\pi} \frac{\varepsilon}{\left(\omega-\bar{E}_{12}\right)^{2}+\varepsilon^{2}} \\
& \times\left|\left\langle\mathbf{p}_{1} \mathbf{p}_{2}\left|T^{R}(\omega+\mathrm{i} \varepsilon)\right| \overline{\mathbf{p}}_{2} \overline{\mathbf{p}}_{1}\right\rangle\right|^{2}\left[\bar{f}_{1} \bar{f}_{2}\left(1 \pm f_{1}\right)\left(1 \pm f_{2}\right)-f_{1} f_{2}\left(1 \pm \bar{f}_{1}\right)\left(1 \pm \bar{f}_{2}\right)\right] .(66
\end{aligned}
$$

The first order retardation contributions from the derivative optical theorem for the T-matrix and the reconstruction formulae used in $I^{0}$ as well as the term $I^{1}$ are collected in $I^{R}$ [21]. Due to the additional term $I^{R}$, the kinetic equation (65) conserves, in contrast to the usual Boltzmann equation, the density and the energy of a nonideal system [21,35].

It is interesting to have a closer look at the term $I^{1}(48)$ in the T-matrix approximation

$$
\begin{aligned}
I^{1}= & \frac{\partial}{\partial T} \int \frac{\mathrm{d} \mathbf{p}_{2}}{(2 \pi)^{3}}\left\{\left.\frac{\partial}{\partial \omega} \operatorname{Re}\left\langle\mathbf{p}_{1} \mathbf{p}_{2}\left|T^{R}(\omega)\right| \mathbf{p}_{2} \mathbf{p}_{1}\right\rangle\right|_{\omega=E\left(\mathbf{p}_{1}\right)+E\left(\mathbf{p}_{2}\right)} f_{1} f_{2}\right. \\
& \left.-\int \frac{\mathrm{d} \omega}{2 \pi}\left\langle\mathbf{p}_{1} \mathbf{p}_{2}\left|T^{<}\left(\omega+\epsilon\left(\mathbf{p}_{2}\right)\right)\right| \mathbf{p}_{2} \mathbf{p}_{1}\right\rangle \frac{P^{\prime}}{\omega-E\left(\mathbf{p}_{1}\right)}\left(1-f_{1}-f_{2}\right)\right\}
\end{aligned}
$$

It is important to remark that the second term in (67) is determined by the off-shell T-matrix. Therefore, it can be expected that this term contains bound state contributions. In order to separate the bound state parts, the off-shell term $T^{<}$is written as a bilinear expansion with respect to the bound and scattering eigenstates of the two-particle Hamiltonian [10,21]

$$
I^{1}=I_{\text {scatt }}^{1}+\frac{\partial}{\partial T} \int \frac{\mathrm{d} \mathbf{p}_{2}}{(2 \pi)^{3}} \sum_{j P}\left\langle\mathbf{p}_{1} \mathbf{p}_{2} \mid j P\right\rangle\left\langle P j \mid \mathbf{p}_{2} \mathbf{p}_{1}\right\rangle N_{j}(P) .
$$


Bound states can be considered now as a new species of composed particles. Then it is obvious to introduce the distribution function of the bound states $f^{B}\left(\mathbf{p}_{1}\right)$ and the free particles by the relations $[21,38]$

$$
\begin{aligned}
f^{B}\left(\mathbf{p}_{1}\right) & =\int \frac{\mathrm{d} \mathbf{p}_{2}}{(2 \pi \hbar)^{3}} \sum_{j P}\left\langle\mathbf{p}_{1} \mathbf{p}_{2} \mid \Psi^{j P}\right\rangle\left\langle\tilde{\Psi}^{j P} \mid \mathbf{p}_{2} \mathbf{p}_{1}\right\rangle N_{j}(P), \\
f\left(\mathbf{p}_{1}\right) & =f^{B}\left(\mathbf{p}_{1}\right)+f^{F}\left(\mathbf{p}_{1}\right) .
\end{aligned}
$$

Using these definitions, we get for the kinetic equation for the free particle distribution function $f^{F}\left(\mathbf{p}_{1}\right)[21,39]$

$$
\left(\frac{\partial}{\partial T}+\frac{\mathbf{p}}{m_{a}} \nabla_{\mathbf{R}}-\nabla_{\mathbf{R}} U_{a}^{\mathrm{eff}}(\mathbf{R}, T) \nabla_{\mathbf{p}}\right) f_{a}^{F}(\mathbf{p}, \mathbf{R}, T)=I^{B}+I_{\mathrm{scatt}}^{R}
$$

This equation is the basic equation of the kinetic theory with reactions, e. g. ionization and recombination, in plasmas. But we have to take into account that the kinetic equation is effected by bound states only if there are changes of the bound state occupation $N_{j}(P)$ with respect to the time. That is the case only in collisions of at least three particles or for external fields. The approximation of the self-energy is therefore to be extended to a cluster expansion up to three or more particles, see e. g. [10,38,40-42].

In summary, we can conclude that the inclusion of the first-order gradient corrections gives an essential generalization of the usual Boltzmann equation. Especially, this equation conserves the total energy in binary collision approximation. Furthermore, in (65) off-shell contributions appear which make it possible to investigate long-living correlations such as bound states.

\section{Acknowledgments}

The authors wish to thank M. Bonitz (Kiel), W.-D. Kraeft and M. Schlanges (Greifswald), P. Lipavský and V. Špička (Prague), C.V. Peletminskij (Kharkov), and K. Henneberger (Rostock) for many fruitful discussions. This work was supported by the Deutsche Forschungsgemeinschaft (SFB 198) and by a grant for CPU time at the HLRZ Jülich.

\section{References}

1. Prigogine I.P., Non-equilibrium Statistical Mechanics. New York, 1962.

2. Resibois P., Physica, 1965, 31, 645.

3. Kadanoff L.P., Baym G. Quantum Statistical Mechanics. New York, 1962

4. Klimontovich Yu.L., Kinetic Theory of Nonideal Gases and Nonideal Plasmas. Moscow, 1975 (in Russian) and Oxford, 1982.

5. Kremp D., Bonitz M., Kraeft W.D., Schlanges M., Ann. Phys. (N.Y.), 1997, 258, 320.

6. Bonitz M., Quantum Kinetic Theory. B. G. Teubner, Stuttgart, Leipzig, 1998.

7. Keldysh L.V., Zh. Eksp. Teor. Fiz., 1964, 47, 1515 [Sov. Phys. - JETP, 1965, 20, 235].

8. Danielewicz P., Ann. Phys. (N.Y.), 1984, 152, 239.

9. Botermans W., Malfliet R., Phys. Rep., 1990, 198, 115.

10. Kremp D., Schlanges M., Kraeft W.D. Quantum Statistics of Nonideal Plasmas. Springer, Berlin, 2005.

11. Wagner M., Phys. Rev. B, 1991, 44, 6104.

12. Morozov V.G., Röpke G, Ann. Phys. (N.Y.), 1999, 278, 127.

13. Fujita S., J. Math. Phys., 1965, 6, 1877.

14. Hall A.G., J. Phys., 1975, 8, 214.

15. Kremp D., Schlanges M., Bornath Th., J. Stat. Phys., 1985, 41, 661.

16. Semkat D., Kremp D., Bonitz M., Phys. Rev. E, 1999, 59, 1557.

17. Semkat D., Kremp D., Bonitz M., J. Math. Phys., 2000, 41, 7458.

18. Kremp D., Semkat D., Bonitz M. In: Progress in Nonequilibrium Green's Functions. Ed. M. Bonitz, World Scientific Publ., Singapore, 2000.

19. Klimontovich Yu.L., Ebeling W., Zh. Eksp. Teor. Fiz., 1972, 63, 905. 
20. Bärwinkel K., Z. Naturf., 1969, 24a, 38.

21. Bornath Th., Kremp D., Kraeft W.D., Schlanges M., Phys. Rev. E, 1996, 54, 3274.

22. Keldysh L.V. In: Progress in Nonequilibrium Green's Functions II. Eds. M. Bonitz and D. Semkat, World Scientific Publ., Singapore, 2003.

23. Martin P.C., Schwinger J., Phys. Rev., 1959, 115, 1342.

24. Semkat D., Bonitz M., Kremp D., Contrib. Plasma Phys., 2003, 43, 321.

25. Bonitz M., Kremp D., Scott D.C., Binder R., Kraeft W.D., Köhler H.S., J. Phys.: Cond. Matter, 1996, 8, 6057.

26. Köhler H.S., Kwong N.H., Yousif H.A., Comp. Phys. Comm., 1999, 123, 123.

27. Köhler H.S., Kwong N.H., Binder R., Semkat D., Bonitz M. In: Progress in Nonequilibrium Green's Functions. Ed. M. Bonitz, World Scientific Publ., Singapore, 2000.

28. Bonitz M., Semkat D. (Eds.). Introduction to Computational Methods in Many-Body Physics. Rinton Press, Paramus, 2006.

29. Gericke D.O., Murillo M.S., Semkat D., Bonitz M., Kremp D., J. Phys. A: Math. Gen., 2003, 36, 6087.

30. Semkat D., Bonitz M., Kremp D., Murillo M.S., Gericke D.O., In: Progress in Nonequilibrium Green's Functions II. Eds. M. Bonitz and D. Semkat, World Scientific Publ., Singapore, 2003.

31. Bonitz M., Semkat D., Murillo M.S., Gericke D.O. In: Progress in Nonequilibrium Green's Functions II. Eds. M. Bonitz and D. Semkat, World Scientific Publ., Singapore, 2003.

32. Lipavský P., Špička V., Velický B., Phys. Rev. B, 1986, 34, 6933.

33. Špička V., Lipavský P., Phys. Rev. Lett., 1994, 73, 3439.

34. Špička V., Lipavský P., Phys. Rev. B, 1995, 52, 14615.

35. Lipavský P., Morawetz K., Špička V., Kinetic Equations for Strongly Interacting Dense Fermi Systems. Annales de Physique, 2001, 26, No. 1.

36. Kremp D., Kraeft W.D., Lambert A.J.M.D., Physica A, 1984, 127, 72.

37. Akhiezer A.T., Peletminskij S.V. Methods of Statistical Physics. Nauka, Moscow, 1977 (in Russian) [Pergamon, Elmsford, N.Y., 1977]

38. Klimontovich Yu.L., Kremp D., Physica A, 1981, 109, 517.

39. Peletminskij S.V., Teor. Mat. Fiz., 1971, 6, 123.

40. Schlanges M., Bornath Th., Contrib. Plasma Phys., 1997, 37, 239.

41. Klimontovich Yu.L., Kremp D., Kraeft W.D., Adv. Chem. Phys., 1987, 58, 175.

42. Kremp D., Schlanges M., Bornath Th. In: The Dynamics of Systems with Chemical Reactions. Ed. J. Popielawski, World Scientific, Singapore, 1989.

\title{
Короткочасова кінетика та початкові кореляції у неідеальних квантових системах
}

\author{
Д.Кремп, Д.Семкат, Т.Борназ \\ Університет Ростоку, Інститут фізики, D-18051 Росток, Німеччина \\ Отримано 16 березня 2006 р., в остаточному вигляді - 12 травня 2006 р.
}

$€$ багато причин щоб розглядати квантові кінетичні рівняння, що описують еволюцію багаточастинкової системи в ультракоротких часових масштабах, наприклад збереження енергії у неідеальній плазмі, наростання кореляцій (зв'язані стани) та кінетика ультрашвидких процесів у короткоімпульсній лазерній плазмі.

Представлено квантову кінетичну теорію, що включає початкові кореляції та є справедливою на довільних часових масштабах. Показано різні приклади короткочасових релаксаційних процесів, такі як релаксація функції розподілу та енергії. Крім того обговорюється градієнтний розклад загальних рівнянь та роль зв'язаних станів.

Ключові слова: Кінетична теорія, дійсночасові функції Гріна, короткочасова релаксація

PACS: $05.20 . \mathrm{Dd}, 52.25 . \mathrm{Dg}, 52.27 . \mathrm{Gr}$ 